\title{
A relação entre sujeitos com transtorno mental e equipamentos sociais
}

\author{
Ana Carolina de Moraes Dantas Moura ${ }^{a}$, Moema Luzia Barros de Mourab \\ Vera Lúcia Dutra Facundesc, Ivo de Andrade Lima Filhoc, \\ Luziana Carvalho de Albuquerque Maranháo ${ }^{c}$, Maria Jucineide Lopes Borges ${ }^{d}$
}

aPrograma de Residência em Saúde da Família, Universidade Federal de Pernambuco - UFPE, Recife, PE, Brasil bPrograma de Pós-graduação em Psicologia Clínica, Universidade Católica de Pernambuco - Unicap, Recife, PE, Brasil ${ }^{\mathrm{c}}$ Curso de Terapia Ocupacional, Universidade Federal de Pernambuco - UFPE, Recife, PE, Brasil

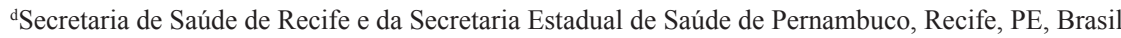

\begin{abstract}
Resumo: O cuidado em saúde mental oferecido atualmente na atenção primária inclui ferramentas que apresentam o território comunitário como um espaço privilegiado para o novo processo de assistência. Nesse sentido, as intervenções junto à população com transtornos mentais devem voltar-se ao desenvolvimento de estratégias que busquem promover mudanças no espaço social ocupado por essas pessoas e à construção e/ou resgate de redes territoriais. Este estudo tem por objetivo conhecer a relação entre os indivíduos com transtorno mental e os equipamentos sociais da comunidade, por meio de investigação qualitativa com sete usuários com história de sofrimento psíquico, assistidos em uma unidade da Estratégia Saúde da Família. Foram identificados 97 equipamentos sociais na comunidade, distribuídos nas seguintes categorias: saúde, religião, educação, lazer, organização social e postos de trabalho. Observou-se que os usuários participavam apenas de atividades religiosas e recorriam à Estratégia Saúde da Família para cuidados de saúde. A escassez de espaços e atividades de lazer na comunidade acentua a dificuldade relativa à participação social desses sujeitos. Conclui-se que os sujeitos apresentaram relações restritas ao núcleo familiar, aos profissionais da saúde da Estratégia Saúde da Família e às atividades religiosas, o que reflete uma articulação frágil com a rede comunitária de cuidados. Esse fato indica a necessidade de expansão das ações de saúde mental na atenção primária e reitera a prática da Terapia Ocupacional, contribuindo para ampliar as relações territoriais e cotidianas de sujeitos com necessidades psicossociais.
\end{abstract}

Palavras-chave: Atenção Primária à Saúde, Saúde Mental, Terapia Ocupacional.

\section{The relationship between subjects with mental disorder and social facilities}

\begin{abstract}
The mental care currently provided in primary health care includes tools which present the community territory as a privileged space for the new assistance process. This way, interventions with the population with mental disorders should be aimed at developing strategies which seek the promotion of changes in the social space occupied by these people and at constructing and/or resuming territorial networks. This study aims to understand the relationship between individuals with mental disorder and the community social facilities by means of a qualitative investigation with 7 users, who present a history of psychic suffering, assisted at a unit of the Family Health Strategy. We identified 97 social facilities in the community, distributed into the following categories: health, religion, education, leisure, social organization, and workplaces. We observed that users only participated in religious activities and they resorted to the Family Health Strategy for health care. The lack of spaces and leisure activities in the community increases the difficulty with regard to the social participation of these subjects. We conclude that
\end{abstract}

Autor para correspondência: Vera Facundes, Departamento de Terapia Ocupacional, Centro de Ciências da Saúde, Universidade Federal de Pernambuco, Av. Prof. Moraes Rego, 1235, Cidade Universitária, CEP 50670-901, Recife, PE, Brasil e-mail: verafacundes@ yahoo.com.br Recebido em 21/9/2012; Revisão em 20/8/2013; Aceito em 16/1/2014. 
the subjects present relationships limited to the nuclear family, health professionals at the Family Health Strategy, and religious activities, reflecting a fragile connection to the community network of care. This fact indicates a need for expanding mental care actions in primary care and reinforces the practice of occupational therapy, contributing to increase the territorial and everyday life relationships of subjects with psychosocial needs.

Keywords: Primary Health Care, Mental Health, Occupational Therapy.

\section{Introdução}

A superação do modelo manicomial proposta pela atenção psicossocial preconiza os princípios da democratização, participação social, corresponsabilização, escuta polifônica, acolhimento e transversalidade. No Brasil, pode-se identificar um conjunto de estratégias e princípios que configura um cenário político propício à participação e construção social de um novo espaço para a saúde mental. Alguns dispositivos têm contribuído para a reorganização da atenção aos sujeitos com transtorno mental, tais como: leis estaduais de reforma psiquiátrica, criação de espaços de participação social e centros de convivência, a Estratégia Saúde da Família (ESF) e iniciativas culturais (AMARANTE, 2007).

O cenário das práticas de saúde mental passa a ser, então, constituído pela articulação entre a ESF, os serviços de saúde mental, como centros de atenção psicossocial (CAPS), hospitais-dia, ambulatórios etc., e os demais dispositivos, como centros de convivência, projetos culturais e de geração de renda etc. Esse modelo representa uma das mais efetivas frentes de atuação do Sistema Único de Saúde (SUS) no contexto da reforma psiquiátrica, compondo, assim, uma rede de cuidado que deve ir além do âmbito do setor da saúde e interagir com outros espaços da cidade para proporcionar um conjunto vivo e concreto de referências às pessoas em sofrimento mental (AMARANTE, 2008; HENNA et al., 2008).

O foco das intervençóes passa a considerar a subjetividade dos sujeitos e suas singularidades, reconhecendo neles possibilidades de relacionar e recriar condiçóes concretas de vida. As intervençóes implicam, também, construir possibilidades reais de sociabilidade, trabalho, moradia, lazer e educação, visando à reinserção dos sujeitos em seu território existencial; esse objetivo pressupóe uma articulação intersetorial entre as políticas públicas, para garantir uma rede sólida de cuidado (ALMEIDA; TREVISAN, 2010; AMARANTE, 2007; BELINI; HIRDES, 2006; BRASIL, 2013).

Sob essa perspectiva, a Atenção Primária à Saúde se apresenta como porta de entrada preferencial do SUS, devendo compor e coordenar a rede de cuidados aos indivíduos, às famílias e aos grupos, inclusive aos cidadãos que demandam cuidados em saúde mental. Como suas açôes são desenvolvidas em território geograficamente conhecido, os profissionais desse nível de atenção à saúde estabelecem uma proximidade estratégica com os usuários para o cuidado em saúde mental, pois há maior possibilidade de identificar a história de vida das pessoas e seus vínculos com a comunidade/território onde vivem, bem como com outros elementos do contexto de vida que singularizam o sujeito em sua inserção sociocultural, buscando, assim, proporcionar uma atenção integral (BRASIL, 2013)

Dessa forma, a intervenção junto à população com transtornos mentais deve ser direcionada ao desenvolvimento de estratégias que busquem promover mudanças no espaço social ocupado por essas pessoas. Fazem-se necessários o fortalecimento das redes sociais e a construção e/ou o resgate de redes territoriais, uma vez que é no território que se desenvolvem os mecanismos de solidariedade, rejeição, fraternidade e discriminação no dia a dia de determinada cultura social (ALMEIDA; TREVISAN, 2010; AMARANTE, 2007; BARROS; MÂNGIA, 2007).

Segundo Fontes (2008), o território remete a um campo de amarração das sociabilidades cotidianas no local onde se vive. Logo, identificar os equipamentos sociais de uma comunidade, entender as relaçóes estabelecidas pelos indivíduos com tais equipamentos e as causas de uma náo relação significa, também, conhecer o território e os sujeitos em suas relaçóes cotidianas.

Esse conhecimento contribui para analisar as necessidades objetivas e subjetivas da população e, consequentemente, amplia a visão de saúde relativa à definição de direitos fundamentais (saúde, lazer, educação, liberdade de expressão, convívio social etc.) (MOREIRA, 2008; BARROS; LOPES; GALHEIGO, 2007).

Diante dessa contextualização teórica, este artigo tem por objetivo conhecer a relação entre os indivíduos com transtorno mental e os equipamentos sociais da comunidade. De modo específico, são descritas as características sociodemográficas dos usuários; são identificados os equipamentos sociais disponíveis nessa comunidade; e é indicada a relação que os usuários estabelecem com tais equipamentos.

Em relação aos postos de trabalho, são identificadas as atividades produtivas dos usuários dos serviços de saúde nesses locais, visando identificar, 
também, a razão da ruptura ou inexistência desse tipo de relação.

\section{Procedimentos metodológicos}

Este estudo, aprovado pelo Comitê de Ética em Pesquisa da Universidade Federal de Pernambuco (UFPE) sob protocolo n. 441/2010 constitui uma investigação qualitativa desenvolvida no âmbito do projeto de pesquisa-ação Terapia Ocupacional na Atenção Básica.

A pesquisa foi realizada na Unidade de Saúde da Família (USF) Sítio Wanderley, localizada na comunidade de Brasilit, bairro da Várzea, pertencente ao Distrito Sanitário IV da cidade de Recife. No período de estudo, essa USF possuía 4.002 famílias cadastradas e estimava-se que a população coberta por ela totalizava 12.212 habitantes. Esse serviço é constituído por três Equipes de Saúde da Família (EqSF); este estudo limitou-se à população atendida pela EqSF 1 .

A amostra consistiu em sete usuários dessa USF com história de sofrimento psíquico, selecionados de modo intencional, por meio de levantamento exploratório. Foram incluídos indivíduos com idade $\geq 18$ anos, com transtorno mental e em situaçáo de risco e/ou com agravos relacionados à saúde mental identificados pelos agentes comunitários de saúde (ACS) da EqSF 1. Foram excluídos os usuários que se encontravam em internamento hospitalar ou em outro tipo de afastamento de sua residência durante a coleta de dados.

Os dados apresentados neste estudo foram coletados por meio de entrevistas com os usuários em seu domicílio ou em espaços da comunidade entre março e maio de 2011. Para isso, foi utilizado um roteiro semiestruturado com questóes relativas ao perfil sociodemográfico, ao reconhecimento e à utilização dos equipamentos sociais disponíveis no território. A análise qualitativa dos dados ocorreu após transcrição, leitura integral e pareamento de conteúdo, segundo os propósitos do estudo, e os resultados foram correlacionados com a literatura científica pertinente ao tema.

Todos os sujeitos receberam informaçóes e explicaçóes acerca da pesquisa e confirmaram sua participação por meio da assinatura do Termo de Consentimento Livre e Esclarecido.

\section{Resultados}

\subsection{Caracterização sociodemográfica dos usuários}

Dos sete usuários que participaram do estudo, seis tinham história de transtorno mental, com percurso de tratamento em hospital psiquiátrico, CAPS e/ou ambulatório de saúde mental (Tabela 1).

Tabela 1. Caracterização sociodemográfica dos usuários com transtorno mental em situações de risco e/ ou com agravos relacionados a problemas de saúde mental, Recife, 2010.

\begin{tabular}{|c|c|c|c|c|c|}
\hline Usuário & Sexo & Idade (anos) & $\begin{array}{l}\text { Situação } \\
\text { conjugal }\end{array}$ & Filhos & Religião \\
\hline A. R.S.S. & F & 37 & Solteiro & 2 & Católica \\
\hline A.S.S. & M & 52 & Solteiro & 0 & Católica \\
\hline E. F. S. & $\mathrm{F}$ & 56 & Viúvo & 6 & Católica \\
\hline I. B. S. & $\mathrm{F}$ & 45 & Viúvo & 1 & Evangélica \\
\hline L. B. S. & M & 49 & Casado & 1 & Evangélica \\
\hline M. F. B. & $\mathrm{F}$ & 49 & Divorciado & 0 & Católica \\
\hline S. B. L. & $\mathrm{F}$ & 40 & Divorciado & 2 & Evangélica \\
\hline Usuário & Escolaridade & $\begin{array}{c}\text { Núcleo } \\
\text { habitacional }\end{array}$ & $\begin{array}{l}\text { Experiência de } \\
\text { trabalho }\end{array}$ & Renda & $\begin{array}{c}\text { Tempo de } \\
\text { residência no } \\
\text { bairro (anos) }\end{array}$ \\
\hline A. R. S. S. & EFI & Família & Vendedora & Aposentadoria & 36 \\
\hline A.S.S. & EFI & Família & Mecânico & Aposentadoria & $>30$ \\
\hline E. F. S. & EFI & Família & $\begin{array}{l}\text { Doméstica em } \\
\text { casa de família }\end{array}$ & Bolsa Família & $>25$ \\
\hline I. B. S. & EFI & Sozinho & Serviços gerais & Aposentadoria & 45 \\
\hline L. B. S. & EFI & Família & $\begin{array}{l}\text { Encarregado de } \\
\quad \text { firma }\end{array}$ & Aposentadoria & 49 \\
\hline M. F. B. & EMC & Família & Professora & Aposentadoria & $>30$ \\
\hline S. B. L. & EMC & Família & Comerciária & Aposentadoria & 40 \\
\hline
\end{tabular}


Não foi possível identificar o diagnóstico desses sujeitos, pois não havia registro desse fato na ficha A de cadastro da USF e os usuários e/ou seus familiares não souberam informá-lo com precisão, embora relatassem doenças como esquizofrenia, depressão e transtorno bipolar. Esses seis usuários utilizavam medicaçôes psicotrópicas e, no período de coleta de dados, mencionaram dificuldades no acompanhamento ambulatorial com o psiquiatra, o que os levou a solicitar ao médico da USF a renovação da receita por longo período, sem avaliação de um especialista em psiquiatria.

O único usuário sem história de transtorno mental, do sexo feminino, foi incluído no estudo porque apresentava sinais de sofrimento psíquico, como isolamento, tristeza, falta de interesse e de disposição para cuidar de si e de sua casa, principalmente depois que contraiu tuberculose e foi afastada de seu emprego. A ACS consultada informou que, segundo a avaliação da médica da USF, não havia indicação de prescrição medicamentosa específica para esses sintomas. Essa usuária foi convidada a participar do grupo de convivência formado na USF como atividade de intervenção do projeto Terapia Ocupacional na Atenção Básica.

A idade dos sete sujeitos deste estudo, cinco do sexo feminino e dois do masculino, variou dos 37 aos 56 anos; cinco indivíduos tinham ao menos um filho e todos já mantiveram relacionamento conjugal em determinada fase da vida, porém, apenas um estava casado no período de realizaçáo da pesquisa.

Quanto à religião, observou-se uma distribuição homogênea entre católicos e evangélicos; em relação à escolaridade, apenas dois indivíduos tinham completado o ensino médio, enquanto os demais não haviam concluído o ensino fundamental. Segundo Ludermir (2008), a educação apresenta um efeito direto sobre a saúde mental, pois aumenta a possibilidade de escolhas na vida da pessoa e influencia as aspirações, a autoestima e a aquisição de conhecimentos, que podem motivar atitudes e comportamentos mais saudáveis. Observa-se, assim, uma associação direta entre a quantidade de anos de estudo e a saúde mental, como constatado neste estudo. Esse achado também pode estar relacionado ao fato de os espaços educacionais não se mostrarem preparados para lidar com pessoas em sofrimento psíquico e de que essas, com frequência, não concluem seus estudos.

Todos os indivíduos relataram experiência com atividade produtiva e, durante o período de realização do estudo, apenas um usuário, do sexo feminino, não se encontrava aposentado por invalidez, porém possuía outra fonte de renda que também não era derivada de trabalho. Sabe-se que os transtornos mentais representam quatro das dez principais causas de incapacidade em todo o mundo e que afetam $25 \%$ da população em alguma fase de sua vida. Tal processo de afastamento temporário e/ou definitivo do trabalho, por qualquer motivo, pode gerar um impacto negativo na vida do indivíduo. Quando esse afastamento decorre de manifestaçóes psicopatológicas e comportamentais de transtorno mental, reforça-se o estigma, o preconceito e a exclusão social, uma vez que o trabalho é considerado, em nossa sociedade, mediador de interação social, construtor de subjetividade, gerador de renda e fonte de troca e construção da rede social do indivíduo (MIRANDA et al., 2009; LUSSI et al., 2011).

Observou-se, ainda, que todos os indivíduos residiam no bairro havia pelo menos 25 anos e dividiam o espaço domiciliar com pelo menos um familiar (pai, mãe, irmão, sobrinho, cônjuge etc.), exceto um usuário, do sexo feminino, que relatou morar sozinho, porém, ao lado da casa de parentes, com os quais tinha contato diariamente. Esse achado corrobora Muramoto e Mângia (2011), uma vez que, em geral, as redes sociais de pessoas com transtornos mentais mostram-se mais reduzidas do que aquelas de pessoas sem transtornos dessa natureza; também se aponta o fato de que, com frequência, essas redes são constituídas por um único tipo de relação, restrito aos laços familiares, caracterizado por falta de reciprocidade de procura e reconhecimento. Não raro, essas redes podem incluir profissionais do serviço de saúde mental.

Dentre os sujeitos da pesquisa prevaleceu o sexo feminino, o status de aposentado e a baixa escolaridade, corroborando outros estudos da literatura pertinente, que apontam alta prevalência de transtornos mentais em mulheres e em indivíduos excluídos do mercado de trabalho formal de baixa renda e baixa escolaridade (LUDERMIR, 2008).

\subsection{Equipamentos sociais disponíveis na comunidade}

O território onde se localiza a USF Sítio Wanderley compreende diversos equipamentos sociais, que foram classificados de acordo com sua finalidade, ou seja, serviços de saúde, instituições de educação e de atividade religiosa, organizaçóes sociais, espaços de lazer e postos de trabalho. A Figura 1 apresenta os dados quantitativos acerca desses equipamentos.

Os serviços de saúde da comunidade são constituídos pela USF Sítio Wanderley, uma equipe do Núcleo de Apoio à Saúde da Família (NASF), além de consultórios médicos e odontológicos privados. A USF conta com açốes de matriciamento 
do CAPS Espaço Vida, o serviço de referência em saúde mental do Distrito Sanitário IV.

Uma diversidade de espaços religiosos, como igrejas evangélica e católica, centro espírita e terreiro de candomblé foi identificada na comunidade. Dentre as instituiçôes de educação encontravam-se escolas de educação infantil, ensino fundamental e ensino médio, sendo apenas duas delas públicas (uma municipal e outra estadual). Constatou-se, ainda, a existência de atividades de organização social, por meio de associação de moradores e clube de mães.

No entanto, os espaços de lazer são escassos na comunidade (lan house e praça pública); quanto aos postos de trabalho, observou-se predominância em relação aos demais equipamentos mencionados, com destaque para o comércio (farmácia, mercado, padaria, bar, restaurante, sorveteria etc.).

Uma vez que a atenção primária prioriza ações voltadas à promoção da saúde e prevenção de agravos no território, é fundamental identificar os equipamentos sociais disponíveis na comunidade com vistas à atenção integral (ANDRADE; BÜCHELE; GEVAERD, 2007). Dessa forma, a EqSF deve estabelecer uma relação de corresponsabilidade não só com os usuários e suas famílias, mas também com os demais setores (educação, cultura, lazer, trabalho, habitação etc.), visando à integralidade e territorialidade da atenção à saúde.

Segundo Fontes (2007), a concepçáo do modelo do sistema de saúde, a partir da reforma sanitária, tem como conteúdo principal a construção e o resgate das redes comunitárias. Pois é por meio dessas redes que o indivíduo entra em contato com seu campo de sociabilidade secundária, isto é, ONGs e grupos religiosos, culturais, educativos e de apoio comunitário, além de estabelecer sua rede de sociabilidade primária, composta por amigos, parentes, colegas de trabalho, vizinhos etc.

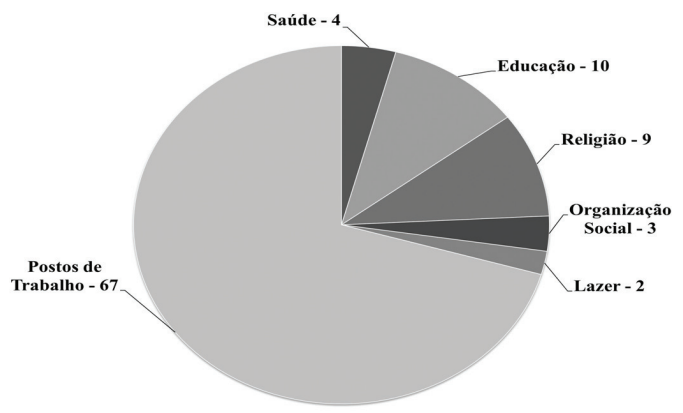

Figura 1. Distribuição dos equipamentos sociais na área coberta pela USF Sítio Wanderley, Recife, 2010.
Assim, atualmente, o cuidado em saúde mental defende a atenção domiciliar, a reconstrução das redes sociais e o empoderamento do sujeito com transtorno mental. Ainda de acordo com Fontes (2007), essas práticas são norteadas pelo padrão apresentado nas diretrizes da reforma sanitária referentes à territorialização, à participação social e ao ambiente multidisciplinar.

\subsection{Relação dos usuários com os equipamentos sociais}

Constatou-se que todos os sujeitos do estudo conheciam pelo menos dois equipamentos sociais disponíveis na comunidade e frequentavam no máximo dois deles (Tabela 2).

Dentre os equipamentos mais conhecidos e frequentados encontravam-se o espaço religioso e o serviço de saúde. A maioria dos usuários frequentava a igreja semanalmente e buscava a USF para renovar a receita dos medicamentos psicotrópicos ou fazer acompanhamento de doenças como hipertensão ou diabetes.

Então, nenhum dos usuários frequentava escola nem participava de atividades de organização social, embora conhecessem alguns desses locais. $\mathrm{A}$ interrupção e o não retorno aos estudos foram atribuídos, pelos indivíduos, à dificuldade de permanecer em sala de aula, ao medo de não aprender ou à sensação de não ter mais condiçôes de retornar aos estudos em decorrência dos sintomas de transtorno mental.

Apenas um dos usuários relatou frequentar a praça, espaço público de lazer da comunidade, e outros três afirmaram conhecer esse local. Além do espaço não oferecer atividades regulares que estimulassem a presença da populaçáo, um usuário afirmou que náo frequentava esse local por ser um ponto constantemente visitado para uso de drogas.

Destaca-se nos achados a ausência de atividades de lazer na vida desses indivíduos, tanto por conta do número reduzido desses espaços como, ainda, por não se observarem açóes de saúde voltadas ao incentivo às atividades de lazer, educacionais e culturais, além da participação social.

A maioria dos usuários informou que frequentava ou já havia frequentado alguma instituição religiosa. Esse achado corrobora Koenig (2007), que trata da relação entre envolvimento religioso e saúde mental. $\mathrm{O}$ autor indica que a maioria das pesquisas em populaçóes saudáveis sugere que as crenças e práticas religiosas estão associadas a maior bem-estar, melhor saúde mental e enfrentamento mais efetivo 
Tabela 2. Utilização dos equipamentos sociais pelos usuários com transtorno mental em situações de risco e/ou com agravos relacionados a problemas de saúde mental, Recife, 2011.

\begin{tabular}{clc}
\hline Usuário & \multicolumn{1}{c}{ Equipamentos que conhece } & Equipamentos que frequenta \\
\hline A. R.S.S. & Igreja, escola e USF & Igreja e USF \\
A.S.S. & Igreja, praça pública e USF & Praça pública \\
E. F.S. & Igrejas, escola pública, praça pública, clube de mães e USF & USF \\
I. B.S. & Igreja, praça pública e USF & Igreja e USF \\
L.B.S. & Igreja, USF & USF \\
M. F. B. & Igreja, escola, clube de mães e USF & Igreja e USF \\
S. B.L. & Igreja e terreiro de candomblé, escola, praça pública, clube & Igreja e USF \\
& de mães, associação de moradores e USF & \\
\hline
\end{tabular}

de situaçóes estressantes; ele aponta, também, a importância de avaliar quando as crenças religiosas ou espirituais são utilizadas para lidar melhor com a doença mental e quando podem exacerbar essa doença.

Observou-se que, apesar da existência de um número significativo de postos de trabalho na comunidade, não foi identificada nenhuma relação produtiva dos usuários nesses locais, embora alguns tenham relatado experiências anteriores de trabalho, inclusive na própria comunidade. Essas atividades foram interrompidas pelas crises decorrentes do transtorno mental e pelo consequente afastamento para realizar o tratamento. Esse fato levou os indivíduos à aposentadoria por invalidez, provocou isolamento social, impossibilitou trocas interpessoais e reduziu laços de amizade, o que repercute em maior exclusão social e torna-os alvo de preconceito.

Estudos apontam que, em média, a rede social de pessoas com transtorno mental é 70\% composta por familiares que coabitam com elas, enquanto esse percentual é de $50 \%$ na população em geral (MURAMOTO; MÂNGIA, 2011). Esse achado corrobora Saraceno (1996), constatando-se que as pessoas com transtornos mentais possuem contratualidade social próxima a zero, uma vez que se encontram afastadas do mercado de trabalho e de atividades culturais, de educação e de lazer e, em consequência, são discriminadas, marginalizadas e excluídas, frequentando, basicamente, a USF e a igreja.

As dificuldades de participaçáo social dos usuários foram abordadas durante a coleta de dados, para discutir as possibilidades de enfrentamento. Os usuários foram convidados a participar do grupo de convivência formado na USF como atividade de intervenção do projeto Terapia Ocupacional na Atenção Básica; seis deles aderiram à proposta, que caracteriza uma pesquisa-ação. Durante a pesquisa, as atividades foram realizadas com o intuito de desenvolver habilidades pessoais, para resgatar o fazer cotidiano no contexto social e cultural desses sujeitos, e visaram à prevenção de agravos à saúde e à melhoria da qualidade de vida.

Castro et al. (2001) acrescentam que, na Terapia Ocupacional, a promoção da saúde visa ao equilíbrio nas atividades de trabalho, lazer, autocuidado e repouso. Entende-se que é por meio dessas atividades que os sujeitos se articulam com sua comunidade, viabilizam a construção social de espaços de vida e de expressão da diversidade. Assim, as ações de saúde mental na atenção primária devem seguir o modelo de redes de cuidado, de base territorial, atuação transversal com outras políticas específicas e que busquem o estabelecimento de vínculos $\mathrm{e}$ acolhimento (BRASIL, 2003).

\section{Considerações finais}

Constatou-se neste estudo uma relaçáo fragilizada entre os usuários da USF com história de sofrimento psíquico e os espaços de lazer, educação, organização social e trabalho disponíveis na comunidade. Tal achado merece destaque devido ao fato de que os sujeitos do estudo relataram residir no bairro há pelo menos 25 anos; esperava-se que os equipamentos sociais do território fossem mais utilizados por eles.

Observou-se menor frequência dos usuários da USF com história de sofrimento psíquico nos locais de lazer, em comparação com as demais categorias de indivíduos. Pode-se indicar que isso se deve à oferta tímida dessas atividades, ao pequeno interesse desses usuários e à ausência de ações de saúde que incentivem essa prática. Pautados no conceito ampliado de saúde, que amparou a criação do SUS, os profissionais da saúde devem valorizar esse aspecto da vida dos sujeitos que, na maioria das vezes, é negligenciado por não se considerar que o lazer também é fundamental em relação à qualidade de vida. 
Constituiu limitação deste estudo a não investigação da utilização, por parte desses usuários, dos serviços disponibilizados pelos postos de trabalho, como farmácia, mercado, bar, restaurante etc. Isso aponta a necessidade de que outros estudos sejam desenvolvidos, para aprofundamento das discussóes apresentadas neste artigo.

Os sujeitos apresentaram relações restritas ao núcleo familiar, aos profissionais da saúde da USF e às atividades religiosas, o que reflete uma articulação frágil com a rede comunitária de cuidados.

Ressalta-se, ainda, a dificuldade vivenciada pelos usuários para realizar acompanhamento especializado de saúde mental e, assim, eles encontram na USF a possibilidade de renovar as receitas de medicamentos psicotrópicos.

Essas questóes apontam a necessidade de ampliarem-se as açôes na atenção primária relativas à saúde mental, como de fortalecimento da articulação entre os serviços de saúde mental e a rede intersetorial de cada município.

Nesse sentido, conhecer como se dão as relaçôes sociais dos indivíduos com transtorno mental no território que habitam e quais fatores nelas interferem é primordial para o planejamento de açóes que minimizem os impactos causados pelo estigma, preconceito e discriminação em relação aos transtornos mentais e que favoreçam a participação dessas pessoas na sociedade.

Esse olhar que se debruça na relação do homem com suas atividades cotidianas, considerando as potencialidades e os limites de cada um, amplia o raciocínio da Terapia Ocupacional. Isso reforça a importância e o compromisso da área no cuidado, uma vez que suas ações favorecem a construção social de espaços de vida e de expressão da diversidade, em consonância com os princípios de promoçẫo da saúde, prevençáo de agravos e reabilitação psicossocial do SUS.

\section{Referências}

ALMEIDA, D. T.; TREVISAN, E. R. Estratégias de intervenção da terapia ocupacional em consonância com as transformaçōes da assistência em saúde mental no Brasil. Interface: Comunicação, Saúde, Educação, Botucatu, v. 15, n. 36, p. 299-307, 2010. Disponível em: $<$ http://www.scielo.br/scielo.php?script=sci_arttext\&p $\mathrm{id}=$ S1414-32832011000100023 >. Acesso em: 12 ago. 2013.

AMARANTE, P. Saúde mental e atenção psicossocial. Rio de Janeiro: Fiocruz, 2007.

AMARANTE, P. Saúde mental, desinstitucionalização e novas estratégias de cuidado. In: GIOVANELLA,
L. et al. (Orgs.). Políticas e sistema de saúde no Brasil. Rio de Janeiro: Fiocruz, 2008. p. 735-759.

ANDRADE, S. R.; BÜCHELE, F.; GEVAERD, D. Salud mental en servicios básicos de salud en Brasil. Enfermería Global, Murcia, v. 6, n. 10, p. 1-11, 2007. Disponível em: $<$ http://revistas.um.es/eglobal/article/view/214>. Acesso em: 12 ago. 2013.

BARROS, D. D.; LOPES, R. E.; GALHEIGO, S. M. Novos espaços, novos sujeitos: a terapia ocupacional no trabalho territorial e comunitário. In: CAVALCANTI, A.; GALVÃO, C. Terapia ocupacional: fundamentação e prática. Rio de Janeiro: Guanabara Koogan, 2007. p. 354-363.

BARROS, J. O.; MÂNGIA, E. F. Rede social e atenção às pessoas com transtornos mentais: novo desafio para os serviços de saúde mental. Revista de Terapia Ocupacional da USP, São Paulo, v. 18, n. 3, p. 135-142, 2007. Disponível em: <http://www.revistas.usp.br/rto/article/view/14017>. Acesso em: 12 ago. 2013.

BELINI, M. G.; HIRDES, A. Projeto Morada São Pedro: da institucionalização à desinstitucionalização em saúde mental. Texto \& Contexto Enfermagem, Florianópolis, v. 15, n. 4, p. 562-569, 2006. Disponível em: <http:// www.scielo.br/pdf/tce/v15n4/v15n4a03.pdf>. Acesso em: 12 ago. 2013.

BRASIL. Ministério da Saúde. Saúde mental e atenção básica: o vínculo e o diálogo necessários. Brasília, 2003. Disponível em: <http://portal.saude.gov.br/portal/arquivos/ pdf/diretrizes.pdf $>$. Acesso em: 12 ago. 2013.

BRASIL. Ministério da Saúde. Secretaria de Atenção à Saúde. Departamento de Atenção Básica. Saúde mental. Brasília, 2013. 176 p. (Cadernos de Atenção Básica, n. 34). Disponível em: <http://189.28.128.100/dab/ docs/portaldab/publicacoes/caderno_34.pdf >. Acesso em: 5 ago. 2013.

CASTRO, E. D. et al. Atividades humanas e terapia ocupacional. In: CARLO, M. M. R. P.; BARTALOTTI, C. C. (Orgs.). Terapia ocupacional no Brasil: fundamentos e perspectivas. São Paulo: Plexus, 2001. p. 41-59.

FONTES, B. A. S. M. Redes sociais e saúde: sobre a formação de redes de apoio social no cotidiano de portadores de transtorno mental. Politica \& Trabalho: Revista de Ciências Sociais, João Pessoa, n. 26, p. 87-104, 2007. Disponível em: <http://periodicos.ufpb. br/ojs/index.php/politicaetrabalho/article/view/6774>. Acesso em: 12 ago. 2013.

FONTES, B. A. S. M. Dos pavilhôes às ruas: a âncora territorial da reforma psiquiátrica. Revista de Terapia Ocupacional da USP, São Paulo, v. 19, n. 3, p. 183-192, 2008. Disponível em: <http://www.revistas. usp.br/rto/article/view/14046>. Acesso em: 12 ago. 2013.

HENNA, E. S. et al. Rede de atenção à saúde mental de base comunitária: a experiência de Santo André. Revista Brasileira de Crescimento e Desenvolvimento Humano, São Paulo, v. 18, n. 1, p. 16-26, 2008. Disponível em: <http://pepsic.bvsalud.org/scielo.php?pid=S0104$12822008000100004 \&$ \&cript $=$ sci_arttext $>$. Acesso em: 12 ago. 2013. 
KOENIG, H. G. Religião, espiritualidade e psiquiatria: uma nova era na atenção à saúde mental. Revista de Psiquiatria Clínica, São Paulo, v. 34, n. 1, p. 5-7, 2007. Suplemento. Disponível em: <http://www.hcnet.usp.br/ ipq/revista/vol34/s1/5.html>. Acesso em: 12 ago. 2013.

LUDERMIR, A. B. Desigualdades de classe e gênero e saúde mental nas cidades. Physis: Revista de Saúde Coletiva, Rio de Janeiro, v. 18, n. 3, p. 451-467, 2008. Disponível em: <http://www.scielo.br/scielo.php?script=sci_artte xt\&pid $=$ S0103-73312008000300005>. Acesso em: 12 ago. 2013.

LUSSI, I. A. O. et al. Reabilitação psicossocial: oficinas de geração de renda no contexto da saúde mental. $O$ Mundo da Saúde, São Paulo, v. 35, n. 2, p. 185-192, 2011. Disponível em: <http://bvsms.saude.gov.br/bvs/artigos/ reabilitacao_psicossocial_oficinas_geracao_renda_saude_ mental.pdf $>$. Acesso em: 12 ago. 2013.

MIRANDA, F. A. N. et al. Saúde mental, trabalho e aposentadoria: focalizando a alienação mental. Revista Brasileira de Enfermagem, Brasília, v. 62, n. 5, p. 711-716, 2009. Disponível em: <http:// www.scielo.br/scielo.php?script $=$ sci_arttext $\&$ p $\mathrm{id}=$ S0034-71672009000500010 >. Acesso em: 12 ago. 2013.

MOREIRA, A. B. Terapia ocupacional: história crítica e abordagens territoriais/comunitárias. Revista Vita et Sanitas, Trindade, v. 2, n. 2, p. 79-91, 2008. Disponível em: <http://www.fug.edu.br/revista_2/pdf/artigo_to.pdf $>$. Acesso em: 12 ago. 2013.

MURAMOTO, M. T.; MÂNGIA, E. F. A sustentabilidade da vida cotidiana: um estudo das redes sociais de usuários de serviço de saúde mental no município de Santo André (SP, Brasil). Ciência \& Saúde Coletiva, Rio de Janeiro, v. 16, n. 4, p. 2165-2177, 2011. Disponível em: <http://www.scielo.br/scielo.php?script=sci_arttext\&p $\mathrm{id}=$ S1413-81232011000400016>. Acesso em: 12 ago. 2013.

SARACENO, B. Reabilitação psicossocial: uma estratégia para a passagem do milênio. In: PITTA, A. M. F. (Org.). Reabilitação psicossocial no Brasil. São Paulo: Hucitec, 1996. p. 13-18.

\section{Contribuição dos Autores}

Todos os autores foram responsáveis pela pesquisa, organização e análise dos resultados, além da redação e revisão do texto. 